\title{
Diseño, traducción y adaptación de una entrevista sociocultural a docentes sobre Aprender a Aprender
}

\author{
Hugo Armando Brito Rivera \\ INVALSI, Roma, Italia. \\ hugobrito.rivera ext亩invalsi.it \\ ORCID: https://orcid.org/0000-0003-1363-5980 \\ Daniela Torti \\ Università di Genova, Genova, Italia. \\ daniela.tortidedu.unige.it \\ ORCID: https://orcid.org/0000-0002-7562-8113
}

Agda Malheiro Ferraz de Carvalho

Fundação Carlos Chagas, São Paulo, Brasil.

agdamfclayahoo.com.br

ORCID: https://orcid.org/0000-0001-9904-0093

\section{RESUMEN}

Se aborda el planteamiento y diseño de una entrevista enfocada en la perspectiva cultural de Aprender a Aprender (AaA) desarrollada como parte de un proyecto coordinado por el Instituto Nacional para la Evaluación del Sistema Educativo y de Instrucción y Formación de Italia y realizado en seis países de Europa (España e Italia) y Latinoamérica (Brasil, Ecuador, México y Uruguay). Se describe desde una aproximación general el proceso de elaboración, traducción y adaptación del instrumento. Este se compuso de nueve núcleos temáticos donde AaA fue perfilado a partir de tres categorías; creación de sentido, afrontar lo nuevo y confianza en sí mismo. La versión final de la entrevista es considerada como un resultado que interpela la compartición de un objeto de estudio multi-local de acuerdo a una perspectiva de investigación intercultural. El estudio pretender identificar la connotación de AaA de acuerdo a la cultura de pertenencia en docentes de estudiantes de 5, 10, 13 y 15 años. Se espera que los resultados beneficien a la investigación educativa aportando evidencia empírica útil para estudios encuadrados en esta perspectiva y contribuyan al diseño de políticas educativas y formación docente a partir de la identificación de buenas prácticas de enseñanza de AaA.

Palabras clave: aprender a aprender, profesorado no universitario, investigación intercultural, entrevista sociocultural, competencias de aprendizaje. 


\section{La perspectiva cultural de Aprender a Aprender}

Aprender a Aprender (AaA) forma parte de las metas de sistemas educativos interesados en la promoción de competencias y habilidades de aprendizaje en estudiantes de todos los niveles escolares. Diversos organismos, locales e internacionales, han reiterado la importancia de enseñar y aprender a aprender, justificando a esta competencia como objetivo de la educación y factor estratégico para el desarrollo económico y bienestar social (cfr. OECD, 2009; UNESCO, 2013). Si bien se trata de un constructo discutido a través de una vasta área de estudios durante los últimos cincuenta años este mantiene su validez para responder a las desafíos de las sociedades contemporáneas (시ello, 2018) tales como la incertidumbre y la rapidez de los cambios tecnológicos, económicos o sociales. La pertinencia de AaA se relaciona con las competencias profesionales y personales requeridas de frente a contextos de innovación educativa y laboral (Álvarez-Arregui, 2019; Arreguit y Hughes, 2019).

Respecto a su definición teórica destaca la dificultad de integrar las corrientes de investigación que se han encargado de su estudio (Deakin Crick, Stringher y Ren, 2014), siendo caracterizada como una competencia compleja y transversal que interpela la capacidad y disposición a aprender de frente a nuevas situaciones y a lo largo de la vida (Claxton, 2002; Marcuccio, 2016). La evolución histórica del constructo incluye su planteamiento en las áreas de la cognición, meta cognición, habilidades de estudio y auto regulación del aprendizaje así como el campo de las características de la personalidad (cfr. Heckman, Stixrud y Urzúa, 2006) y transferencia de AaA de un contexto a otro (particularmente entre escuela y trabajo) (cfr. Tuomi-Gröhn y Engeström, 2003).

En el año 2006 la Comisión Europea incluyó AaA entre las competencias clave para el aprendizaje permanente, definiéndola como "la habilidad para iniciar el aprendizaje y persistir en él, para organizar su propio aprendizaje y gestionar el tiempo y la información eficazmente, ya sea individualmente o en grupos. Esta competencia conlleva ser consciente del propio proceso de aprendizaje y de las necesidades de aprendizaje de cada uno, determinar las oportunidades disponibles y ser capaz de superar los obstáculos con el fin de culminar el aprendizaje con éxito" (Comisión Europea, 2006, p. 16). Recientemente, en el año 2018, AaA fue incluido nuevamente como parte de las competencias clave (Comisión Europea, 2018) siendo conceptualizado en esta ocasión como parte de la "competencia personal, social y de aprender a aprender", cuya orientación general recae en el ámbito de las relaciones interpersonales y la resolución de las propias necesidades de aprendizaje.

Aunque AaA ha sido señalado persistentemente como una competencia fundamental para continuar aprendiendo, activando en ello procesos y dinámicas en torno a la sociedad del aprendizaje y del conocimiento, también es evidente que la historia de esta competencia se vincula con estudios y políticas educativas influidas principalmente por culturas anglosajonas (e.g. Estados Unidos o Inglaterra) (Deakin Crick, Stringher y Ren, 2014). En el presente artículo sostenemos que el conjunto de diferencias que AaA adquiere de acuerdo a la cultura de pertenencia ha sido considerado en menor medida en el debate educativo predominante. Este a su vez se adscribe habitualmente a la tradición del pensamiento "occidental".

Un ejemplo de ello son las investigaciones en torno al diagnóstico y evaluación de AaA en contextos escolares. Tales experiencias se circunscriben mayoritariamente al ámbito europeo (Gargallo, Pérez, García, Giménez y Portillo, 2020) y consisten en evaluar el AaA en estudiantes por medio de la medición de sus componentes, particularmente de carácter afectivo, cognitivo y meta cognitivo. Tal es el caso del inventario de aprendizaje permanente y eficaz desarrollado en Inglaterra (Effective Lifelong Learning Inventory) (Deakin Crick, Broadfoot, y Claxton, 2004), la prueba de competencias transversales realizada en Holanda (Cross-Curricular Skills Test) (Elshout-Mohr, Meijer, Oostdam y Van Gelderen, 2004), la escala propuesta por Hautamäki et al., (2002) en Finlandia o el propio test para evaluar AaA llevado a cabo en el Centre for Research on Education and Lifelong Learning de la Comisión Europea (Gargallo et al., 2020). Entre los cuestionamientos a estos instrumentos se encuentra el de su pertinencia en relación a otros contextos, incluyendo en ello la discusión acerca de la connotación que AaA puede tener en otras culturas.

El conocimiento de tal connotación cultural involucra la dimensión general del sentido de educar y la función que las escuelas adquieren en la formación de esta competencia, considerada la más antigua de los sistemas escolares (Ajello y Torti, 2019). Desde este punto de vista AaA requiere ser ubicado en la línea de continuidad de la reflexión pedagógica y filosófica sobre el sentido de la propia instrucción educativa, entendida como selección de contenidos y memoria colectiva que se pretende formar en las nuevas generaciones. El enfoque cultural de AaA conlleva, por lo tanto, considerar las diferencias entre las distintas maneras de concebir la educación y las creencias tácitas o explícitas que se articulan en torno al proceso de enseñanza y aprendizaje de acuerdo con la cultura de pertenencia (Bruner, 1996; Rogoff, 1990). El aprendizaje es ponderado en este sentido como un fenómeno cultural e histórico asociado con contextos escolarizados, informales o laborales (Hager y Halliday, 2006).

Desde esta perspectiva toda institución educativa tiende a desarrollar en los estudiantes capacidades intelectuales que son consideradas relevantes para resolver determinados problemas y crear productos que son consecuentemente valorados en la propia cultura (Gardner, 1983). Retomando la discusión de Vygotsky (1986) respecto a la relación entre instrucción y desarrollo de facultades superiores del pensamiento, destaca la función de "disciplina formal" que las materias escolares tienen en la formación de competencias y habilidades que serán utilizadas por los estudiantes en el futuro. La enseñanza de las materias escolares (ejemplificadas por Vygotsky en el latín y griego) sobresale por su valor para generar competencias de pensamiento y aprendizaje que serán útiles para afrontar situaciones dentro y fuera del contexto escolar. En este orden de ideas se sostiene que los docentes eligen contenidos y habilidades a formar en los estudiantes al considerarlos fundamentales para generar el desarrollo de, a su vez, otras habilidades y competencias (i.e. no solamente para transmitir el contenido curricular). Desde este punto de vista enseñar a AaA está presente implícitamente en la elección de las propuestas didácticas -y concepciones asociadas a éstas- que los docentes realizan en la actividad escolar cotidiana.

Tal planteo representa parte del presupuesto de partida para indagar el AaA como una competencia que moviliza conocimientos, emociones y valores en relación al ambiente social y cultural de pertenencia. Puesto que el planteamiento aquí expuesto constituye un campo de investigación por ser plenamente desarrollado este trabajo se posiciona como un paso intermedio en esta dirección. El objetivo del artículo consiste en presentar una aproximación general de carácter descriptivo al proceso de diseño, traducción y adaptación de una entrevista llevada a cabo como parte de un proyecto internacional de investigación centrado en la perspectiva cultural de AaA. Sostenemos que la contribución de un enfoque investigativo de este tipo consiste, entre otros aspectos, en abordar las posibilidades de integración entre el discurso educativo "globalizante" y la diversidad de las 
realidades locales a fin de proponer definiciones y metodologías de formación coherentes con las poblaciones y contextos donde se pretende su desarrollo.

\section{El proyecto de investigación coordinado por INVALSI}

En el año 2015, tras un ejercicio de autoevaluación, las escuelas italianas evidenciaron la necesidad de recibir apoyo de INVALSI para el desarrollo y evaluación de la competencia de AaA (incluida en el currículo nacional). Docentes del sistema educativo italiano señalaron dificultades para implementar experiencias escolares centradas en la promoción de esta competencia.

La demanda inicial de tales centros escolares condujo a INVALSI, hacia el año 2017, a estructurar un proyecto de investigación enfocado en las potenciales dificultades, semejanzas o diferencias que el desarrollo de AaA encontraría en sistemas escolares de países que compartieran determinados rasgos y atributos. La etapa inicial del proyecto se integró de una reseña sistemática sobre AaA en la literatura internacional y el análisis de currículos y sistemas educativos, enfocándose originalmente pero no de modo exclusivo en el caso latinoamericano (Patera, 2018; Stringher, Di Rienzo, Brito, Davis y García, 2019).

Posteriormente, se proyectó una fase de investigación cualitativa con el objetivo de indagar la presencia y naturaleza de AaA en la práctica escolar de distintos contextos. Esta fase ha tenido como objetivo conocer la connotación cultural de AaA y comprender cómo se adquiere en diferentes sistemas educativos. Se convocó la participación de equipos de investigación en Brasil (Fundação Carlos Chagas), Ecuador (Universidad Politécnica Salesiana), España (Universidad de Cantabria), México (Universidad Autónoma Metropolitana - Iztapalapa) y Uruguay (Instituto Nacional de Evaluación Educativa y Universidad de la República), además del caso italiano (INVALSI), con un total de 20 investigadores.

Los sistemas educativos de los países participantes incluyen en mayor o menor medida, directa o indirectamente, aspectos asociados a la competencia de AaA. Al mismo tiempo tales países se ubican geopolíticamente en demarcaciones no anglosajonas relativas a la noción de "sur del mundo" (Santos, 2011), encontrándose en una posición periférica respecto al discurso educativo "globalizante" vinculado con esta competencia. Algunos rasgos compartidos por estos países son la heterogeneidad cultural (i.e. profundas diferencias al interior de una misma realidad nacional), brechas sociales y educativas, así como las desigualdades y asimetrías regionales (i.e. acceso a oportunidades educativas), sociales y económicas. Tales aspectos impactan la adquisición y desarrollo de la competencia en cuestión.

La fase cualitativa, de naturaleza empírica, se centró en la aplicación de una entrevista semi estructurada a docentes de estudiantes de 5, 10, 13 y 15 años en las áreas de lengua y ciencias sociales y matemáticas y ciencias (a excepción de los docentes de niños de 5 años). El diseño de la guía de entrevista tuvo lugar a inicio del año 2018. En términos generales, la etapa cualitativa se sustentó en un cuadro teórico y metodológico descriptivo, comparativo e interpretativo, no tributario de una concepción "comparativista" en el uso de instrumentos calibrados en contextos culturales radicalmente diferentes.

Tratándose de un objeto educativo con presencia latente o manifiesta en la historia de los sistemas escolares de países latinoamericanos y europeos durante las últimas décadas, AaA puede significar, más que una realidad educativa, una expresión propia al ámbito de la deseabilidad social. Un aspecto emergente consistió por lo tanto en responder cómo indagar la connotación cultural de AaA evitando la deseabilidad social y las etiquetas profesionales presentes en el discurso educativo. Se señaló en consecuencia la importancia de acceder al punto de vista de los participantes por medio de la expresión de respuestas auténticas.

La entrevista semi estructurada se eligió como instrumento y estrategia de investigación idónea para indagar el AaA desde el punto de vista personal y cultural de los docentes (Kvale, 2011; Serranò y Fasulo, 2011; Szymanski, Almeida y Prandini, 2004). Este instrumento se consideró útil para hacer emerger el mapa mental de los participantes y poner en evidencia la representación de AaA. Tal representación se considera como un medio de conexión entre lenguaje y cultura, posible a través de la interpretación conjunta de la realidad e intercambio entre entrevistador y entrevistado.

A tal propósito, se consideraron las implicaciones dialógicas y simbólicas derivadas del uso de una entrevista. De acuerdo con Serranò y Fasulo (2011) la entrevista permite acceder a los sentidos y significados asociados a las prácticas cotidianas (Aguiar y Davis, 2011) favoreciendo la emergencia de aquello que tiene valor para los entrevistados y por lo tanto integra su discurso. Este instrumento fue elegido por su pertinencia para captar la connotación cultural observable en la explicación mediante la que los docentes fundamentan las razones por las cuales realizan determinadas prácticas (Bruner, 1990).

\section{El diseño de la entrevista}

La entrevista fue diseñada con el objetivo de reconstruir la práctica cotidiana de los docentes teniendo en cuenta aspectos indicativos de la competencia de AaA. El primer paso para la construcción de la entrevista consistió en la identificación de tres categorías clave a partir de un riguroso análisis teórico: creación de sentido, afrontar lo nuevo y confianza en sí mismo (Hautamäki y Kupiainen, 2014; Deakin Crick, Broadfoot y Claxton, 2004; Stringher, 2014). Dado que AaA refiere al individuo que actúa autónomamente, se consideró fundamental reconstruir las dimensiones que influyen en la confianza en las propias habilidades de aprendizaje y compromiso para implicarse y orientarse en las asignaturas escolares. Cada categoría interpela el AaA en función del siguiente planteamiento:

- Creación de sentido alude a la participación en actividades auténticas mediante las que se estimula y preserva el interés y motivación de aprender. Se sostiene que tanto la continuidad del aprendizaje como la posibilidad de aprender en modo permanente se ven reforzados mediante actividades escolares con un alto sentido para el aprendiz. Se trata asimismo de una función de la competencia de AaA pues ésta conduce a su vez a la creación de sentido de la realidad de quien aprende (Stringher, 2014).

- Afrontar lo nuevo refiere a la asociación entre AaA y la capacidad de resiliencia para enfrentar circunstancias nuevas o adversas de aprendizaje. Con esta categoría se apela al concepto perspectiva de esperanza como elemento clave para continuar aprendiendo. De acuerdo con Hautamäki y Kupiainen (2014, p.177) la perspectiva de esperanza involucra la facilitación o inhibición de fuerzas motivacionales y afectivas que influyen en la iniciativa y disposición para explorar una nueva situación de aprendizaje. Tal acción involucra creencias y actitudes relacionadas con el apoyo o bloqueo del deseo de continuar aprendiendo ya sea dentro de un área disciplinar o respecto a nuevas situaciones de aprendizaje. Esta categoría implica el daño a la motivación o incompetencia aprendida que pueden producirse a través de experiencias escolares negativas (Ajello y Torti, 2019). 
- Confianza en sí mismo interpela el sentido de auto eficacia y confianza en la propia capacidad de aprendizaje. Esta categoría involucra las creencias y actitudes relacionadas con el sujeto que aprende. La confianza en sí mismo orienta la competencia de AaA por medio del componente afectivo (Deakin Crick, Broadfoot y Claxton, 2004; cfr. Hautamäki y Kupiainen, 2014) y tiene repercusión a través de situaciones en las que se fomenta la responsabilidad, la toma de decisiones y la participación del aprendiz.

Debido a la complejidad y número de componentes asociados con AaA (Stringher, 2014) la identificación de tales categorías permitió la simplificación conceptual de esta competencia a fin de orientar la construcción de la entrevista.

Posteriormente se solicitó un coloquio con una profesora italiana para obtener un "testimonio privilegiado" (Losito, 1988) que, debido a la experiencia y trayectoria profesional de la participante, ayudara a reconstruir la actividad docente cotidiana y a identificar las áreas temáticas de la entrevista. El resultado de esta estrategia, basada en un enfoque emico y hermenéutico (Trinchero, 2002; Montesperelli, 1998), condujo a la definición de diez temáticas con las que el equipo INVALSI elaboró subsiguientemente la primera versión en italiano de la guía de entrevista. Esta se compuso de 28 preguntas que fueron formuladas en referencia a cada temática por medio de un lenguaje coloquial a fin evitar respuestas influidas por la deseabilidad social o el uso de etiquetas profesionales.

El siguiente paso consistió en el pre-pilotaje de ocho entrevistas en Italia (con docentes del nivel infancia, primaria y secundaria) y dos más en México (con docentes de primaria y secundaria) en base a una primera traducción del instrumento. En ambos casos el propósito consistió en evaluar la pertinencia del instrumento para obtener información vinculada con la práctica cotidiana y verificar la presencia o ausencia de aspectos asociados con las categorías teóricas antes mencionadas. En esta fase las entrevistas fueron conducidas a través de dos estrategias:

- Entrevista directa. Correspondió al modo habitual de realizar una entrevista y consistió en solicitar al docente la respuesta inmediata a las preguntas, siempre de acuerdo a su propia experiencia y punto de vista.

- Entrevista indirecta. Se inspiró parcialmente en la técnica de "entrevista sobre una entrevista" (Mauceri, 2018) y consistió en solicitar al participante retroalimentación acerca de cómo funcionaría la guía si fuera aplicada con otros colegas. Se consideró que esta estrategia contribuiría a reducir la percepción de sentirse evaluado o juzgado por el entrevistador profundizando en los motivos por los que, si se consideraba necesario, se proponían modificaciones a las preguntas. Con ello se esperaba activar "indirectamente" respuestas auténticas a las preguntas de la guía por parte del participante.

Una vez obtenidos los resultados del pre-pilotaje se procedió a la etapa de revisión. Se excluyeron preguntas mediante las que se obtuvieron respuestas que no referían a la práctica cotidiana, o temáticas que no arrojaron información relevante de acuerdo con los objetivos de la investigación. En base a tales ajustes se integró nuevamente en lengua italiana la versión que constituiría la base para la traducción y adecuación local del instrumento y se definió un protocolo donde se propuso a los equipos de investigación realizar ambas modalidades de entrevista (directa e indirecta). La versión obtenida de este paso, cuyas temáticas se preservaron hasta la versión final (tabla 1), consistió en una entrevista compuesta del siguiente modo:

Tabla 1.

Núcleos temáticos y descripción mínima; versión final de la entrevista.

\begin{tabular}{ll}
\hline Núcleo temático & Descripción mínima \\
\hline 1. Diferencias & Se requiere al participante reflexionar acerca de ta- \\
entre estudiantes & $\begin{array}{l}\text { les diferencias a través de ejemplos concretos. La } \\
\text { finalidad es la de identificar actividades realizadas }\end{array}$ \\
anteriores & $\begin{array}{l}\text { en consideración de las características y exigencias } \\
\text { de aprendizaje de los estudiantes actuales. }\end{array}$
\end{tabular}

Se solicita al docente indicar actividades cotidianas que involucran en mayor medida a los estu2. Actividades diantes y señalar actividades que no logran invoque involucran a lucrarlos. La intención de esta temática consiste en los estudiantes destacar actividades "con sentido" que contribuyen a activar la motivación e implicación de los estudiantes.

Se solicita al docente compartir su experiencia acerca de un estudiante que tuviera dificultades para involucrarse en actividades escolares pero que posteriormente logró superarlas, ejemplifi-

3. Caso positivo cando las acciones realizadas para ayudar al estudiante. La finalidad consiste en activar aspectos vinculados con la "perspectiva de esperanza" y capacidad para enfrentar dificultades de aprendizaje.

A manera de contrapunto de la temática anterior

4. Caso negativo se requiere al participante la descripción de un caso "imposible" donde no se logró involucrar al estudiante en las actividades escolares cotidianas.

Se pide al docente señalar, desde una perspectiva general, habilidades, competencias u otros atri-

5. Características de los estudiantes que preanuncian éxito o fracaso

6. Evaluación de los estudiantes

aprendiendo

7. Diferencias entre clases escolares del mismo nivel butos asociados al aprendizaje, capacidades socioemocionales y de resiliencia presentes durante el transcurso de la vida. El propósito consiste en comprender la representación del éxito / fracaso y su vínculo con específicas características de los estudiantes de acuerdo con el punto de vista docente.

Se requiere al participante ejemplos de situaciones que le permiten comprender cuando los estudiantes han entendido algún contenido de la clase, así como los instrumentos de evaluación que, debido al convencimiento acerca de su utilidad, son los mayormente utilizados (incluyendo el uso que se da a las calificaciones). Se considera que la comprensión de los estudiantes es fundamental para posibilitar que estos continúen aprendiendo, siendo el objetivo de esta temática comprender la función de la evaluación en un sentido amplio.

La intención de esta temática es la de hacer emerger la representación docente acerca de las cualidades del aprendizaje en grupo a partir de las características que favorecen o no el trabajo en clase.

Se solicita reflexionar acerca del rol de la escuela para enseñar a los estudiantes a continuar aprendiendo, requiriendo ejemplos de actividades dirigidas en este sentido. El objetivo reside en conocer la representación acerca de la eficacia o potencial que la intervención de la escuela pueda tener para favorecer el continuar aprendiendo.

9. Aprender Se requiere a los participantes una definición exa aprender plícita de AaA (a fin de explorar los componentes (explícito) asociados a este) y reflexión acerca de su diferencia respecto al "aprendizaje". 


\section{Traducción y adaptación cultural}

El debate metodológico en torno a estudios que involucran participantes de diversas culturas y lenguas señala que los resultados de una investigación pueden verse afectados por la traducción y adecuación del instrumento de investigación (Harkness et al., 2016). La traducción de un instrumento y su adaptación representa un aspecto fundamental en investigaciones cuya muestra es internacional, multi-cultural y multi-regional. Por esta razón el proyecto aquí referido consideró la discusión acerca de cómo un instrumento construido en función del contexto italiano podía ser adaptado para otros grupos culturales.

La versión original de la guía fue traducida a dos lenguas target; español y portugués, involucrando diferencias y propiedades lingüísticas de los países participantes. La traducción de la lengua de partida a las lenguas target conformó la primera parte del proceso de adaptación cultural. La construcción de las versiones locales involucró a todos los equipos de investigación mediante los siguientes aspectos:

- Traducción de la lengua italiana a las lenguas española y portuguesa: Esta acción implicó calibrar la traducción preliminar al español e involucró el punto de vista experto de los investigadores, varios de estos de competencia bilingüe (español-italiano, italiano-español, portugués-español).

- Discusión del sentido y planteamiento del objeto explorado (perspectiva cultural de AaA).

- Preservación de equivalencia semántica e intención de cada pregunta.

- Adaptabilidad de la guía por medio de formulaciones lingüísticas idóneas entre versiones del español hablado en Ecuador, España, México y Uruguay, y en su caso del portugués hablado en Brasil.

- Adecuación de las preguntas al lenguaje educativo local. Si bien diversos estudios señalan la existencia de una brecha entre metodologías cualitativas y cuantitativas en lo que respecta a la traducción de instrumentos de investigación (Van Nes, Abma, Jonsson y Deeg, 2010) en el presente caso se consideraron estrategias provenientes de ambos modelos. Inicialmente se realizó una analogía con los procedimientos de instrumentos estandarizados, considerando prácticas de traducción asociadas con las características multi-culturales y comparativas de estudios internacionales (Harkness et al., 2016).

De acuerdo a estos la dificultad de traducir un instrumento cuya lengua original no es la del país donde será aplicado requiere la adecuación funcional y operativa del mismo, a fin de alcanzar la equivalencia semántica entre el instrumento original y el instrumento target. De acuerdo con Harkness et al. (2016) la consecución de tal equivalencia es un aspecto clave cuando los reactivos han sido diseñados en relación un determinado contexto y se requiere su adaptación para otras poblaciones. Tal situación implica conservar las propiedades del instrumento original así como el sentido e intención de las preguntas que lo integran.

La búsqueda y consecución de tal equivalencia dio lugar a un procedimiento híbrido guiado por aspectos generales de las etapas planteadas por Harkness et al. (2016) de las cuales la equivalencia constituye un factor transversal; diseño, traducción, pre-prueba y definición final del instrumento. Sin embargo, discutiendo la lógica planteada por estos autores, tales etapas no se limitaron a la realización de una réplica de la guía original en italiano en culturas y lenguas diferentes. La orientación, en cambio, consistió en desarrollar una versión compartida de la entrevista, razón por la cual se incluyó la participación de los investigadores locales en diversos momentos del proceso.

Los elementos considerados de los procedimientos de adaptación y traducción de instrumentos cuantitativos (utilizados como ejes sensibilizantes) se integraron en función de un proceso multi-lateral, creativo y multilingüe de acuerdo a una metodología de traducción multi-céntrica alineada a la naturaleza cualitativa de la entrevista (Larkin, Dierckx de Casterlé y Schotsmans, 2007; Roth, 2013). En esta metodología se destacan roles adicionales al habitual como investigador (i.e. investigador-traductor e investigador-revisor) a partir del desarrollo de la investigación en múltiples contextos lingüístico-culturales.

En base a tal modelo el proceso de traducción, revisión y adaptación se realizó "en espiral" y a medida en que las distintas etapas del pre-piloteo y piloteo se llevaron a cabo. Reflexionar sobre una realidad común para asimismo constituirla fue el objetivo de esta fase, considerando que la construcción de una versión culturalmente adaptada debía corresponder al trabajo conjunto de los equipos internacionales, quienes revisaron y adecuaron las distintas versiones a través de dos fases.

La primera involucró encuentros presenciales de formación y discusión entre el equipo INVALSI y los investigadores de Ecuador, Uruguay y Brasil en sus respectivas sedes durante septiembre del 2018. Los investigadores de México y España se incorporaron al proyecto en el año 2019 por lo que se consideró una segunda fase a través de encuentros virtuales. En todos los casos la interacción entre investigadores tuvo como fin desarrollar la versión local del instrumento y compartir su marco metodológico a partir de la revisión de la traducción y respectiva adaptación.

La entrevista tuvo adaptaciones lingüísticas menores en relación al español hablado en Ecuador, Uruguay, México y España, principalmente vinculadas con el estilo y redacción de las preguntas de acuerdo a las variantes idiomáticas de cada país. Las modificaciones se realizaron directamente en la lengua target y se adscribieron principalmente a la forma de dirigirse al entrevistado; en España y Uruguay se optó por un tratamiento de "tú" ("vos" de acuerdo al uso lingüístico rioplatense) ya que se consideró que así la realización de la entrevista favorecería una relación más cercana y menos formal, posibilitando que los participantes se expresaran con mayor libertad y confianza. En el caso de Ecuador y México se mantuvo el tratamiento de "usted" al entrevistado en concordancia con la cultura docente local. No se realizaron cambios de fondo respecto a la sintaxis y terminología utilizada. Respecto a este último rubro destaca que la guía original se caracterizaba por el empleo de términos no profesionalizantes y cotidianos a fin de no estimular el discurso docente "institucionalizado".

Mientras los equipos en Ecuador, España, México y Uruguay no añadieron nuevas preguntas, en Brasil se propuso una versión modificada con la inserción de nuevas preguntas (vid. tabla 2). En Brasil se infirió que, dado que AaA no es una competencia ampliamente conocida, estudiada o difundida en este país, sería necesario agregar preguntas que permitieran saber qué entendían los entrevistados sobre la nueva legislación brasileña (Base Curricular Nacional Común) y los procesos de enseñanza-aprendizaje basados en el desarrollo de habilidades y competencias.

Un último aspecto que rebasa el ámbito de la traducción pero cuya pertinencia se ubica en el ámbito de la adaptación cultural se relaciona con la aceptación o rechazo de las modalidades directa e indirecta de la entrevista por parte de los equipos de investigación. El equipo brasileño señaló que la modalidad indirecta no sería necesaria, siendo la relación directa entre entrevistador y entrevistado el recurso idóneo para la construcción 
del dato empírico en Brasil. En Ecuador y Uruguay se discutió que tal modalidad podría tener implicaciones de carácter ético debido a su potencial interpretación como ocultamiento al entrevistado acerca de la naturaleza de su participación.

\section{Aspectos generales del estudio piloto}

Los equipos de Ecuador, Uruguay, Brasil e Italia realizaron el pilotaje durante el final del 2018 y primer cuatrimestre 2019, mientras que los equipos de España y México lo llevaron a cabo durante el 2019. La modalidad indirecta fue puesta a prueba en Ecuador, Uruguay e Italia; en todos los países se preservaron las garantías de cumplimiento ético por medio del consentimiento informado. En Brasil el primer piloto se dedicó a la guía de entrevista común (guía uno), aplicando posteriormente la propia versión modificada (guía dos). Se realizaron en total 42 entrevistas piloto (tabla 2).

El pilotaje se llevó a cabo de acuerdo a la siguiente distribución geográfica: Brasil; escuelas públicas y privadas, en el estado de São Paulo, municipios Santo André y São Paulo capital (éste último para la guía uno y dos). Ecuador; escuelas Salesianas de carácter fiscomisional-privadas de la ciudad de Cuenca. España; escuelas públicas y privadas de Cantabria. Italia; escuelas públicas de Roma y sur de Italia. México; escuelas públicas de la Ciudad de México y zona conurbada. Uruguay; escuelas públicas y privadas de Montevideo.

El principal resultado de esta fase consistió en el acuerdo de la versión definitiva para el estudio principal, integrada por 21 preguntas y 11 recursos de profundización (e.g. ¿Qué conside- ra usted que responderían otros docentes?) en versión trilingüe. Tres ejemplos de tales preguntas se presentan en la tabla 3.

Los resultados obtenidos se analizaron por medio de encuentros colectivos virtuales, identificando categorías preliminares emergentes. Tal análisis consintió la identificación de aspectos críticos acerca de la guía, desempeño por parte de los investigadores y funcionamiento de la entrevista a nivel local, entre estos el empleo de la modalidad directa e indirecta; en Ecuador y Uruguay se reportó no percibir diferencias significativas de las respuestas obtenidas entre ambas estrategias.

En Ecuador se observó que la modalidad indirecta no favorecía la proximidad con los entrevistados, requerida en este contexto. En cambio, de acuerdo a los resultados obtenidos en Italia, esta misma estrategia permitió recolectar respuestas más amplias, evitando distorsiones por parte de un investigador externo al contexto escolar. Se decidió sin embargo descartar tal modalidad (por lo que no fue replicada en España y México) en base a dos criterios:

- No contribuía a obtener respuestas comparables entre países. Siendo similar a una conversación informal, no siempre fue previsible el nivel de profundidad en base a los argumentos afrontados por el entrevistador.

- Los entrevistados tendían a responder directamente las preguntas, sin expresar valoraciones acerca de la guía.

Un aspecto sobresaliente del pilotaje atañe al modo en que los propios investigadores, en su lectura y análisis de los resultados preliminares, observaron e interpretaron la competencia de

Tabla 2

Número de entrevistas del estudio piloto y distribución por país

\begin{tabular}{|c|c|c|c|c|c|c|c|c|}
\hline \multirow{2}{*}{ Docentes de estudiantes de: } & \multicolumn{2}{|c|}{ Brasil } & \multirow{2}{*}{ Ecuador } & \multirow{2}{*}{ España } & \multirow{2}{*}{ Italia } & \multirow{2}{*}{ México } & \multirow{2}{*}{ Uruguay } & \multirow{2}{*}{ Total: } \\
\hline & Guía uno & Guía dos & & & & & & \\
\hline 5 años & $1 \mathrm{ED}$ & $4 \mathrm{ED}$ & $\begin{array}{l}1 \mathrm{EI} \\
1 \mathrm{ED}\end{array}$ & & $1 \mathrm{EI}$ & $1 \mathrm{ED}$ & $\begin{array}{c}1 \mathrm{EI} \\
2 \mathrm{ED}\end{array}$ & 12 \\
\hline 10 años & $1 \mathrm{ED}$ & $4 \mathrm{ED}$ & $1 \mathrm{ED}$ & $1 \mathrm{ED}$ & $\begin{array}{c}2 \mathrm{ED} \\
3 \mathrm{EI}\end{array}$ & & $\begin{array}{c}1 \mathrm{EI} \\
1 \mathrm{ED}\end{array}$ & 14 \\
\hline 13 años & & & & & $\begin{array}{c}2 \mathrm{ED} \\
1 \mathrm{EI}\end{array}$ & & & 3 \\
\hline 15 años & $1 \mathrm{ED}$ & $4 \mathrm{ED}$ & $\begin{array}{c}1 \mathrm{EI} \\
1 \mathrm{ED}\end{array}$ & $1 \mathrm{ED}$ & $\begin{array}{c}1 \mathrm{ED} \\
1 \mathrm{EI}\end{array}$ & $1 \mathrm{ED}$ & $\begin{array}{c}1 \mathrm{EI} \\
1 \mathrm{ED}\end{array}$ & 13 \\
\hline Total: & 3 & 12 & 5 & 2 & 11 & 2 & 7 & 42 \\
\hline
\end{tabular}

Nota: EI= Entrevista Indirecta; ED= Entrevista Directa.

Tabla 3.

Versión trilingüe de la entrevista final (preguntas 9, 16 y 17)

\begin{tabular}{|c|c|c|c|}
\hline Núcleo temático: & Italiano: & Español: & Portugués: \\
\hline $\begin{array}{l}\text { Características de los } \\
\text { estudiantes que prea- } \\
\text { nuncian éxito o fraca- } \\
\text { so (pregunta 9) }\end{array}$ & $\begin{array}{l}\text { Con il suo occhio da docente, quali } \\
\text { sono le caratteristiche di uno studente } \\
\text { che secondo lei preannunciano succes- } \\
\text { so o insuccesso in futuro? }\end{array}$ & $\begin{array}{l}\text { De acuerdo con tu mirada docente, } \\
\text { ¿cuáles son las características de un } \\
\text { estudiante que anuncian el éxito o el } \\
\text { fracaso en el futuro? }\end{array}$ & $\begin{array}{l}\text { De acordo com seu "olhar docente", } \\
\text { quais são as características que indi- } \\
\text { cam se um aluno terá sucesso ou fra- } \\
\text { casso, no futuro? }\end{array}$ \\
\hline $\begin{array}{l}\text { Continuar aprendien- } \\
\text { do } \\
\text { (pregunta 16) }\end{array}$ & $\begin{array}{l}\text { Secondo lei che cosa è necessario fare } \\
\text { in modo che gli studenti continuino ad } \\
\text { imparare? }\end{array}$ & $\begin{array}{l}\text { En tu opinión, ¿qué crees que es nece- } \\
\text { sario para garantizar que los alumnos } \\
\text { continúen aprendiendo? }\end{array}$ & $\begin{array}{l}\text { De acordo com você, o que é necessá- } \\
\text { rio fazer para que os alunos continuem } \\
\text { aprendendo? }\end{array}$ \\
\hline $\begin{array}{l}\text { AaA (explícito) } \\
\text { (pregunta 17) }\end{array}$ & $\begin{array}{l}\text { Se dovesse sintetizzarlo in qualche } \\
\text { modo, come caratterizzerebbe lei l'ap- } \\
\text { prendere ad apprendere, l'imparare a } \\
\text { imparare? }\end{array}$ & $\begin{array}{l}\text { Si tuvieras que sintetizarlo de algu- } \\
\text { na manera, ¿cómo caracterizarías el } \\
\text { aprender a aprender? }\end{array}$ & $\begin{array}{l}\text { Se você tivesse que de alguma maneira } \\
\text { sintetizar o aprender a aprender, como } \\
\text { você o faria? }\end{array}$ \\
\hline
\end{tabular}

Nota: Español hablado en España y portugués de Brasil. 
AaA de acuerdo con la perspectiva cultural. De esta situación se desprende que captar tal representación no sea una tarea fácil; el reconocimiento de los rasgos culturales de AaA por parte del investigador (o entre investigadores) y consecuente discusión juega un papel central.

En este tenor las categorías propuestas (creación de sentido, afrontar lo nuevo y confianza en sí mismo), al evocar aspectos teóricos específicos, podrían o no integrar parte de la mirada analítica de los investigadores, pues estos poseen diversos posicionamientos al respecto. Tal diversidad es considerada inherente a una investigación de naturaleza cualitativa e intercultural (Creswell, 2016). Las interpretaciones provistas por los investigadores en torno a la guía representan concepciones asociadas a la cultura de pertenencia, enfoque teórico y propia noción de AaA. Más que ser evitadas tales interpretaciones han sido fundamentales en la discusión y construcción de un objeto de estudio y metodología comunes.

\section{Aspectos conclusivos}

Las principales aportaciones del presente artículo consisten en el planteamiento de la perspectiva cultural de AaA y presentación de la estructura temática de la entrevista. Ambos aspectos representan innovación no solamente respecto a cómo obtener datos que permitan estructurar el mapa cultural en torno a esta competencia; significa asimismo un marco investigativo complementario al planteamiento de los estudios predominantes sobre AaA.

La definición de un instrumento común para los seis países representa a su vez el logro de entendimiento y trabajo coordinado entre investigadores. A través de ello ha sido posible entrecruzar el repertorio de prácticas de investigación de los equipos participantes a partir de la colaboración en el estudio. Por otra parte, consideramos que el desarrollo del proyecto y consecuente sistematización de datos tienen el potencial para beneficiar a la investigación educativa en los siguientes aspectos:

- Identificación y difusión de prácticas docentes en torno a la enseñanza de AaA desde un punto de vista cultural y cotidiano.
- Apoyo a la formación docente y diseño de políticas educativas para desarrollar la competencia de AaA desde una perspectiva coherente con la propia cultura.

- Construcción de definiciones y metodologías adecuadas a los contextos educativos donde se pretende formar esta competencia.

- Desarrollo de otros instrumentos de investigación enfocados en el conocimiento de la connotación cultural de AaA.

Dentro de las limitaciones del artículo es necesario considerar la breve descripción de la entrevista y respectivas temáticas. Derivado de ello las restricciones asociadas con el alcance del instrumento no han sido abordadas. En este sentido destaca que los límites del trabajo se adhieren al ámbito de una aproximación general en torno al proceso de diseño, traducción y adaptación de la entrevista. Se considera necesario discutir a profundidad tales aspectos en alguna otra sede.

Los siguientes pasos apuntan al análisis de los datos del estudio principal y consolidación del trabajo internacional entre países participantes. A mediano plazo se espera que los datos esclarezcan componentes por medio de los que AaA se caracteriza culturalmente, extendiendo la potencial aplicación de la entrevista a otros contextos educativos. Las consideraciones finales de este artículo enfatizan la necesidad de invertir esfuerzos y recursos en investigaciones de esta índole, implicando la experticia y conocimiento de investigadores en diferentes países. Esto contribuirá al intercambio profesional y creará oportunidades de discusión y descubrimiento a partir de problemáticas comunes en cada realidad educativa, promoviendo en ello la identificación e inclusión de sus singularidades y especificidades.

\section{Agradecimientos.}

Agradecemos a Anna Maria Ajello por su contribución en el diseño de la entrevista, a Cristina Stringher por la coordinación del proyecto y a Ana Castro y María Huerta por su revisión del artículo e invaluable apoyo. Especial agradecimiento a todos los investigadores participantes. 


\title{
Design, translation and adaptation of a socio-cultural interview with teachers on learning to learn
}

\author{
Hugo Armando Brito Rivera \\ INVALSI, Roma, Italia. \\ hugobrito.rivera ext@invalsi.it \\ ORCID: https://orcid.org/0000-0003-1363-5980 \\ Daniela Torti \\ Università di Genova, Genova, Italia. \\ daniela.tortidedu.unige.it \\ ORCID: https://orcid.org/0000-0002-7562-8133
}

Agda Malheiro Ferraz de Carvalho

Fundação Carlos Chagas, São Paulo, Brasil.

agdamfclayahoo.com.br

ORCID: https://orcid.org/0000-0002-7562-8113

\begin{abstract}
We addressed the design of an interview focused on the cultural perspective of learning to learn (L2L), developed as part of a project coordinated by the Italian National Institute for Educational Evaluation of Instruction and Training and carried out in six European and Latin American countries (Spain and Italy, Brazil, Ecuador, Mexico and Uruguay). The process of elaboration, translation and adaptation of the instrument is described from a general approach. This was made up of nine thematic nuclei where L2L was outlined from three categories: sense making, coping with the uncertainty and self-confidence. The final version of the interview is considered as a result that challenges the sharing of a multi-local study object according to an intercultural research perspective. The study tries to identify the connotation of L2L according to the culture of belonging in teachers of students of 5, 10, 13 and 15 years. The results are expected to benefit the educational research by providing useful empirical evidence for studies framed in this perspective and contribute to the design of educational policies and teacher training based on the identification of good teaching practices of L2L.
\end{abstract}

Key words: Learning to learn, non-university teachers, intercultural research, socio-cultural interview, learning skills 


\section{The cultural perspective of Learning to Learn}

Learning to Learn (L2L) is part of the goals of educational systems interested in promoting learning competencies and skills in students at all school levels. Various organizations, local and international, have reiterated the importance of teaching and learning to learn, justifying this competence as an objective of education and a strategic factor for economic development and social welfare (cf. OECD, 2009; UNESCO, 2013). Although it is a construct discussed through a vast area of studies during the last fifty years, it maintains its validity to respond to the challenges of contemporary societies (Ajello, 2018) such as the uncertainty and rapidity of technological, economic or social changes. The relevance of L2L is related to the professional and personal competences required in contexts of educational and work innovation (Álvarez-Arregui, 2019; Arreguit \& Hughes, 2019).

Regarding its theoretical definition, it strikes the difficulty of integrating the research pathways that have been in charge of its study (Deakin Crick, Stringher \& Ren, 2014), being characterized as a complex and transversal competence that challenges the ability and willingness to learn in face of new situations and throughout life (Claxton, 2002; Marcuccio, 2016). The historical evolution of the construct includes its approach in the areas of cognition, metacognition, study skills and self-regulation of learning, as well as the field of personality characteristics (cf. Heckman, Stixrud \& Urzúa, 2006) and transfer of L2L from one context to another (particularly between school and work) (cf. Tuomi-Gröhn \& Engeström, 2003).

In 2006 the European Commission included L2L among the key competences for lifelong learning, defining it as "the ability to start learning and persist in it, to organize one's own learning and manage time and information effectively, either individually or in groups. This competence involves being aware of own learning process and the learning needs of each one, determining the available opportunities and being able to overcome obstacles in order to successfully complete learning" (European Commission, 2006, p. 16). Recently, in the year 2018, L2L was included again as part of the key competences (European Commission, 2018), being conceptualized on this occasion as part of the "personal, social and learning to learn competence", whose general orientation lies with the scope of interpersonal relationships and the resolution of one's own learning needs.

Although L2L has been persistently pointed out as a fundamental competence to continue learning, activating in it processes and dynamics around the learning and knowledge society, it is also evident that the history of this competence is linked to educational studies and policies influenced mainly by Anglo-Saxon cultures (e.g. United States or England) (Deakin Crick, Stringher \& Ren, 2014). In this article we argue that the set of diffferences that L2L acquieres to the culture of belonging has been less considered in the predominant educational debate. This, in turn, is usually ascribed to the tradition of "western" thought.

An example of this is the investigations around the diagnosis and evaluation of L2L in school contexts. Such experiences are mostly limited to the European sphere (Gargallo, Pérez, García, Giménez \& Portillo, 2020) and consist of evaluating the L2L in students by measuring its components, particularly of an affective, cognitive and meta-cognitive nature. Such is the case of the effective and lifelong learning inventory developed in England (Deakin Crick, Broadfoot, \& Claxton, 2004), the cross-curricular skills test carried out in the Netherlands (Elshout- Mohr, Meijer, Oostdam, \& Van Gelderen, 2004), the scale proposed by Hautamäki et al., (2002) in Finland or the L2L test itself conducted by the Center for Research on Education and Lifelong Learning from the European Commission (Gargallo et al., 2020). Among the questionings to these instruments is that of their relevance in relation to other contexts, including discussion about the connotation that L2L may have in other cultures.

Knowledge of such a cultural connotation involves the general dimension of the sense of educating and the role that schools acquire in the formation of this competence, considered the oldest of the school systems (Ajello Torti, 2019). From this point of view, L2L needs to be located in the line of continuity of the pedagogical and philosophical reflection on the meaning of educational instruction, which is understood as a selection of content and collective memory that is intended to be formed in the new generations. Therefore, the cultural approach of L2L involves considering the differences between the different ways of conceiving education and the tacit or explicit beliefs that are articulated around the teaching and learning process of each culture (Bruner, 1996; Rogoff, 1990). Learning is weighted in this sense as a cultural and historical phenomenon associated with school, informal or work contexts (Hager Halliday, 2006).

From this perspective, every educational institution tends to develop among students intellectual capacities that are considered relevant to solve certain problems and create products that are consequently valued in their own culture (Gardner, 1983). Returning to Vygotsky's (1986) discussion regarding the relationship between instruction and development of higher faculties of thought, he highlights the role of "formal discipline" that school subjects have in the formation of competencies and skills that will be used by students in the future. The teaching of school subjects (exemplified by Vygotsky in Latin and Greek) stands out for its value in generating thinking and learning skills that will be useful in dealing with situations inside and outside the school context. In this order of ideas, it is maintained that teachers choose content and skills to be formed in students by considering them essential to generate the development of, in turn, other skills and competences (i.e. not only to transmit curricular content). From this point of view, teaching L2L is implicitly present in the choice of didactic proposals and associated conceptions - that teachers carry out in daily school activities.

Such an approach represents part of the starting assumption to investigate L2L as a competence that mobilizes knowledge, emotions and values in relation to the social and cultural environment of belonging. Since the approach here exposed constitutes a field of investigation to be fully developed, this work is positioned as an intermediate step in this direction. The objective of the article is to present a general descriptive approach to the design, translation and adaptation process of an interview carried out as part of an international research project focused on the cultural perspective of L2L. We maintain that the contribution of a research approach of this type consists, among other aspects, in addressing the possibilities of integration between the "globalizing" educational discourse and the diversity of local realities in order to propose definitions and training methodologies consistent with the populations and contexts where L2L development is intended.

\section{The research project coordinated by INVALSI}

In 2015, after a self-assessment exercise, Italian schools showed the need to receive support from INVALSI for the development and evaluation of L2L competence (included in the national curriculum). Teachers from the Italian educational system pointed out difficulties in implementing school experiences focused on promoting this competence. 
The initial demand for such schools led INVALSI, towards the year 2017, to structure a research project focused on the potential difficulties, similarities or differences that the development of L2L would encounter in school systems in countries that share certain traits and attributes. The initial stage of the project was made up of a systematic review of L2L in international literature and the analysis of curricula and educational systems, focusing originally, but not exclusively, on the Latin American case (Patera, 2018; Stringher, Di Rienzo, Brito, Davis \& García, 2019).

Subsequently, a qualitative research phase was projected with the aim of investigating the presence and nature of L2L in school practice in different contexts. The objective of this phase was to learn the cultural connotation of L2L and understand how it is acquired in different educational systems. The participation of research teams in Brazil (Fundação Carlos Chagas), Ecuador (Universidad Politécnica Salesiana), Spain (Universidad de Cantabria), Mexico (Universidad Autónoma Metropolitana-Iztapalapa) and Uruguay (Instituto Nacional de Evaluación Educativa and Universidad de la República), was summoned to the Italian case (INVALSI), with a total of 20 researchers.

The educational systems of the participating countries include, to a greater or lesser extent, directly or indirectly, aspects associated with L2L competence. At the same time, these countries are geo-politically located in non-Anglo-Saxon demarcations relative to the notion of "the south of the world" (antos, 2011), being in a peripheral position regarding the "globalizing" educational discourse related to this competence. Some features shared by these countries are cultural heterogeneity (i.e. profound differences within the same national reality), social and educational gaps, as well as regional, social and economic inequalities and asymmetries (i.e. access to educational opportunities). Such aspects impact the acquisition and development of the competence in question.

The qualitative phase, of empirical nature, focused on the application of a semi-structured interview to teachers of students aged 5,10, 13 and 15 in the areas of language and social sciences and mathematics and science (with the exception of teachers of 5-year-old children). The design of the interview guide took place at the beginning of 2018. In general terms, the qualitative stage was based on a descriptive, comparative and interpretive theoretical and methodological table, not subject of a "comparative" conception in the use of calibrated instruments in radically different cultural contexts.

Being an educational object with a latent or manifest presence in the history of the school systems of Latin American and European countries during the last decades, L2L can mean more than an educational reality, an expression proper to the field of social desirability. An emerging aspect consisted of answering how to investigate the cultural connotation of L2L avoiding the social desirability and professional labels present in educational discourse. Consequently, the importance of accessing the participants' point of view through the expression of authentic responses was pointed out.

The semi-structured interview was chosen as the ideal instrument and research strategy to investigate the L2L from the personal and cultural point of view of the teachers (Kvale, 2011; Serranò \& Fasulo, 2011; Szymanski, Almeida \& Prandini, 2004). This instrument was considered useful to bring up the participants' mental map and highlight the representation of L2L. Such representation is considered as a means of connection between language and culture, possible through the joint interpretation of reality and exchange between interviewer and interviewee.

For this purpose, the dialogical and symbolic implications derived from the use of an interview were considered. Accord- ing to Serranò \& Fasulo (2011), the interview allows access to the meanings associated with daily practices (Aguiar Davis, 2011), favoring the emergence of what has value for the interviewees and therefore integrates their discourse. This instrument was chosen for its relevance to capture the observable cultural connotation in the explanation by which teachers base the reasons why they carry out certain practices (Bruner, 1990).

\section{The design of the interview}

The interview was designed with the objective of reconstructing the daily practice of teachers and taking into account indicative aspects of L2L competence. The first step in the construction of the interview consisted of identifying three key categories from a rigorous theoretical analysis: sense making, coping with the uncertainty and self-confidence (Hautamäki \& Kupiainen, 2014; Deakin Crick, Broadfoot \& Claxton, 2004; Stringher, 2014). Given that L2L refers to the individual who acts autonomously, it was considered essential to reconstruct the dimensions that influence confidence in their own learning skills and commitment to get involved and orient themselves in school subjects. Each category questions the L2L based on the following approach:

- Sense making refers to participation in authentic activities through which the interest and motivation to learn is stimulated and preserved. It is argued that both, the continuity of learning and the possibility of learning in a permanent way, are reinforced by school activities with a high sense for the learner. It is also a function of L2L's competence, since this in turn leads to the creation of a sense of reality for the learner (Stringher, 2014).

- Coping with the uncertainty refers to the association between L2L and resilience to cope with new or adverse learning circumstances. This category appeals to the concept of perspective of hope as a key element to continue learning. According to Hautamäki \& Kupiainen (2014, p.177) the prospect of hope involves the facilitation or inhibition of motivational and affective forces that influence the initiative and willingness to explore a new learning situation. Such action involves beliefs and attitudes related to supporting or blocking the desire to continue learning, either within a disciplinary area or regarding new learning situations. This category implies the damage to motivation or learned incompetence that can occur through negative school experiences (Ajello \& Torti, 2019).

- Self-confidence challenges the sense of self-efficacy and confidence in one's ability to learn. This category involves beliefs and attitudes related to the learning subject. Self-confidence guides the L2L competence through the affective component (Deakin Crick, Broadfoot \& Claxton, 2004; cfr. Hautamäki \& Kupiainen, 2014) and has repercussions through situations in which learner's responsibility, decision-making and participation are fostered.

Due to the complexity and number of components associated with L2L (Stringher, 2014), the identification of the categories mentioned above allowed the conceptual simplification of this competence in order to guide the construction of the interview.

Subsequently, a colloquium was requested with an Italian teacher to obtain a "privileged testimony" (Losito, 1988) that, due to the participant's experience and professional career, helped to reconstruct the daily teaching activity and identify the thematic areas of the interview. The result of this strategy, based 
on an emic and hermeneutical approach (Trinchero, 2002; Montesperelli, 1998), led to the definition of ten themes with which the INVALSI team subsequently developed the first Italian version of the interview guide. This was made up of 28 questions that were formulated in reference to each subject through colloquial language in order to avoid responses influenced by social desirability or the use of professional labels.

The next step consisted of pre-piloting eight interviews in Italy (with teachers of preschool, primary and secondary levels) and two more in Mexico (with teachers of primary and secondary levels) based on a first translation of the instrument. In both cases, the purpose was to assess the relevance of the instrument to obtain information related to daily practice and verify the presence or absence of aspects associated with the afore-mentioned theoretical categories. In this phase, the interviews were conducted through two strategies:

- Direct interview: It corresponded to the usual way of conducting an interview and consisted of asking the teacher for the immediate answer to the questions, always according to their own experience and point of view.

- Indirect interview: It was partially inspired by the technique of "interview on an interview" (Mauceri, 2018) and consisted of asking the participant for feedback on how the guide would work if it were applied with other colleagues. This strategy was considered to contribute to reducing the perception of feeling evaluated or judged by the interviewer; and to deepening the reasons why, if deemed necessary, modifications were proposed to the questions. With this, it was hoped to "indirectly" activate authentic responses to the guide's questions by the participant.

Once the results of the pre-piloting were obtained, the revision stage was carried out. Questions which answers did not refer to daily practice, or which topics did not yield relevant information according to the objectives of the research were excluded. Based on such adjustments, the version that would constitute the basis for the translation and local adaptation of the instrument was integrated into the Italian language again and a protocol was defined where the research teams were proposed to carry out both interview modalities (direct and indirect). The version obtained from this step, with the themes that were preserved until the final version (Table 1), consisted of an interview composed as follows:

Table 1.

Thematic nuclei and minimal description; final version of the interview.

\begin{tabular}{ll}
\hline Thematic core & Minimal description \\
\hline $\begin{array}{l}\text { 1. Differences } \\
\text { between current } \\
\text { and previous } \\
\text { students }\end{array}$ & $\begin{array}{l}\text { The participant is required to reflect on such } \\
\text { purpose is to identify activities carried out in } \\
\text { consideration of the characteristics and learning } \\
\text { demands of current students. }\end{array}$
\end{tabular}

The teacher is asked to indicate daily activities

2. Activities that that involve students to a greater extent and to indicate activities that do not involve them. The the most theme is to point out "meaning ful" activities that help activate students' motivation and involvement.

The teacher is asked to share his experience about a student who had difficulties to be involved in school activities but who later man3. Positive case aged to overcome them, exemplifying the actions taken to help the student. The aim is to activate aspects related to the "perspective of hope" and ability to face learning difficulties.

4. Negative case ticipant is required to describe an "impossible"
case where the student was not able to be in-
volved in daily school activities.
volved in daily school activities.

$\begin{array}{ll} & \begin{array}{l}\text { The teacher is asked to point out, from a general } \\ \text { perspective, skills, competences or other attri- }\end{array} \\ \begin{array}{l}\text { 5. Students' } \\ \text { butes associated with learning, socio-emotion- } \\ \text { that announce }\end{array} & \begin{array}{l}\text { al capacities and resilience present during the } \\ \text { course of life. The purpose is to understand the } \\ \text { representation of success / failure and its link }\end{array} \\ \text { with specific characteristics of the students ac- } \\ \text { cording to the teaching point of view. }\end{array}$

The participants are required to provide examples of situations that allow them to understand when students have understood some content of the class, as well as the assessment instru6. Students' ments that, due to the conviction about their evaluation usefulness, are the most widely used (including the use given to the qualifications). The understanding of students is considered essential to enable them to continue learning. The objective of this theme is to understand the function of evaluation in a broad sense.

\begin{tabular}{ll}
$\begin{array}{l}\text { 7. Differences } \\
\text { between school } \\
\text { groups at the } \\
\text { same educational } \\
\text { level }\end{array}$ & $\begin{array}{l}\text { The intention of this theme is to make emerge } \\
\text { the teaching representation about the qualities } \\
\text { of grournor learning from the characteristics that }\end{array}$ \\
\hline
\end{tabular}

The interviewee is requested to reflect on school's role in teaching students to continue learning, requiring examples of activities di8. Keep learning rected in this regard. The objective is to know the representation about the effectiveness or the potential that the intervention of the school may have to favor continued learning.

Participants are required to give an explicit

9. Learning to learn (explicit) definition of L2L (in order to explore the components associated with it) and a reflection on the difference with "learning".

\section{Translation and cultural adaptation}

The methodological debate around studies involving participants from different cultures and languages indicates that the results of an investigation may be affected by the translation and adequacy of the research instrument (Harkness et al., 2016). The translation of an instrument and its adaptation represents a fundamental aspect in investigations whose sample is international, multi-cultural and multi-regional. For this reason, the project considered the discussion about how an instrument built according to the Italian context could be adapted to other cultural groups. 
The original version of the guide was translated into two target languages; Spanish and Portuguese, accounting for differences and linguistic properties of the participating countries. The translation from the source language to the target languages formed the first part of the process of cultural adaptation. All research teams were involved in the construction of the local versions through the following aspects:

- Translation from Italian to Spanish and Portuguese: This action involved calibrating the preliminary translation into Spanish and involved the expert point of view of the researchers, several of whom are bilingual (Spanish-Italian, Italian-Spanish, Portuguese-Spanish).

- Discussion of the meaning and approach of the explored object (cultural perspective of L2L).

- Preservation of semantic equivalence and intention of each question.

- Adaptability of the guide by means of suitable linguistic formulations between versions of the Spanish spoken in Ecuador, Spain, Mexico and Uruguay, and Portuguese spoken, in case of Brazil.

- Adaptation of the questions to the local educational language.

Although various studies indicate the existence of a gap between qualitative and quantitative methodologies with regard to the translation of research instruments (van Nes, Abma, Jonsson and Deeg, 2010), in the present case, strategies from both models were considered. Initially, an analogy was made with standardized instrument procedures, considering translation practices associated with the multi-cultural and comparative characteristics of international studies (Harkness et al., 2016).

According to the aforementioned studies, the difficulty of translating an instrument whose original language is not that of the country where it will be applied requires its functional and operational adaptation, in order to achieve semantic equivalence between the original instrument and the target instrument. According to Harkness et al. (2016) the achievement of such equivalence is a key aspect when the items have been designed in relation to a certain context and their adaptation is required for other populations. Such a situation implies preserving the properties of the original instrument as well as the meaning and intention of the questions that comprise it.

The search and achievement of such equivalence gave rise to a hybrid procedure guided by general aspects of the stages proposed by Harkness et al. (2016) of which equivalence constitutes a transversal factor; design, translation, pre-test and final definition of the instrument. However, discussing the logic raised by these authors, such stages were not limited to the realization of a replica of the original guide in Italian in different cultures and languages. Instead, the orientation consisted of developing a shared version of the interview, reason for which the participation of local researchers at various points in the process was included.

The elements considered of the adaptation and translation procedures of quantitative instruments (used as briefing axes) were integrated based on a multi-lateral, creative and multilingual process according to a multi-centric translation methodology aligned to the qualitative nature of the interview (Larkin, Dierckx de Casterlé \& Schotsmans, 2007; Roth, 2013). In this methodology, additional roles are highlighted to those usual as researcher (i.e. researcher-translator and researcher-reviewer) from the development of research in multiple linguistic-cultural contexts.
Based on such a model, the translation, revision and adaptation process were conducted "in a spiral" and as the different stages of pre-piloting and piloting were carried out. Reflecting on a common reality to constitute it was the objective of this phase, considering that the construction of a culturally adapted version should correspond to the joint work of international teams, who reviewed and adapted the different versions through two phases.

The first phase involved face-to-face training and discussion meetings between the INVALSI team and researchers from Ecuador, Uruguay and Brazil at their respective headquarters in September 2018. Researchers from Mexico and Spain joined the project in 2019, considered a second phase, with discussions and training taking place through virtual meetings. In all cases, the interaction between researchers was aimed at developing the local version of the instrument and sharing its methodological framework based on the revision of the translation and the respective adaptation.

The interview had minor linguistic adaptations in relation to the Spanish spoken in Ecuador, Uruguay, Mexico and Spain, mainly related to the style and wording of the questions according to the language variations of each country. The modifications were made directly in the target language and were mainly ascribed to the way of addressing the interviewee; in Spain and Uruguay, "tú" treatment was chosen ("vos" according to the language used in the River Plate area) since it was considered that, in this way, conducting the interview would favor a closer and less formal relationship, allowing participants to express themselves with greater freedom and trust. In the case of Ecuador and Mexico, the treatment of "usted" to the interviewee was maintained in accordance with the local teaching culture. No substantive changes were made regarding the syntax and terminology used. Regarding this last item, it is worth noting that the original guide was characterized by the use of non-professionalizing and everyday terms in order not to stimulate the "institutionalized" teaching discourse.

While the teams in Ecuador, Spain, Mexico and Uruguay did not add new questions, in Brazil a modified version was proposed with the insertion of new questions (see table 2). In Brazil it was inferred that, since L2L is not a widely known competence, studied or disseminated in this country, it would be necessary to add questions that would allow knowing what interviewees understood about the new Brazilian legislation (Common National Curricular Base) and the teaching processes -learning based on the development of skills and competences.

A last aspect that goes beyond the field of translation but whose relevance is located in the field of cultural adaptation is related to the acceptance or rejection of the direct and indirect modalities of the interview by the research teams. The Brazilian team pointed out that the indirect modality would not be necessary, the direct relationship between the interviewer and the interviewee was considered the ideal source for the construction of the empirical data in Brazil. In Ecuador and Uruguay, it was discussed that such modality could have ethical implications due to its potential interpretation as concealment to the interviewee about the nature of their participation.

\section{General aspects of the pilot study}

The teams from Ecuador, Uruguay, Brazil and Italy carried out the piloting during the end of 2018 and the first four months of 2019, while the teams from Spain and Mexico carried it out during 2019. The indirect modality was tested in Ecuador, Uruguay and Italy. In all countries, guarantees of ethical compliance 
Table 2.

Number of pilot study interviews by country

\begin{tabular}{|c|c|c|c|c|c|c|c|c|}
\hline \multirow[b]{2}{*}{ Teachers from student age } & \multicolumn{2}{|c|}{ Brazil } & \multirow[b]{2}{*}{ Ecuador } & \multirow[b]{2}{*}{ Spain } & \multirow[b]{2}{*}{ Italy } & \multirow[b]{2}{*}{ Mexico } & \multirow[b]{2}{*}{ Uruguay } & \multirow[b]{2}{*}{ Total: } \\
\hline & $\begin{array}{c}\text { Guide } \\
1\end{array}$ & Guide 2 & & & & & & \\
\hline 5 years old & $1 \mathrm{DI}$ & $4 \mathrm{DI}$ & $\begin{array}{c}1 \mathrm{II} \\
1 \mathrm{DI}\end{array}$ & & $1 \mathrm{II}$ & $1 \mathrm{DI}$ & $\begin{array}{c}1 \mathrm{II} \\
2 \mathrm{DI}\end{array}$ & 12 \\
\hline 10 years old & $1 \mathrm{DI}$ & $4 \mathrm{DI}$ & $1 \mathrm{DI}$ & $1 \mathrm{DI}$ & $\begin{array}{c}2 \mathrm{DI} \\
3 \mathrm{II}\end{array}$ & & $\begin{array}{c}1 \mathrm{II} \\
1 \mathrm{DI}\end{array}$ & 14 \\
\hline 13 years old & & & & & $\begin{array}{c}2 \mathrm{DI} \\
1 \mathrm{II} \\
\end{array}$ & & & 3 \\
\hline 15 years old & $1 \mathrm{DI}$ & $4 \mathrm{DI}$ & $\begin{array}{c}1 \mathrm{II} \\
1 \mathrm{DI}\end{array}$ & $1 \mathrm{DI}$ & $\begin{array}{c}1 \mathrm{DI} \\
1 \mathrm{II}\end{array}$ & $1 \mathrm{DI}$ & $\begin{array}{c}1 \mathrm{II} \\
1 \mathrm{DI}\end{array}$ & 13 \\
\hline Total: & 3 & 12 & 5 & 2 & 11 & 2 & 7 & 42 \\
\hline
\end{tabular}

Note: $\mathrm{II}=$ Indirect Interview; DI= Direct Interview.

were preserved through informed consent. In Brazil, the first pilot study was devoted to the common interview guide (guide one), subsequently applying the modified version (guide two). A total of 42 pilot interviews were conducted (Table 2).

The piloting was carried out according to the following geographical distribution: Brazil: public and private schools, in the state of São Paulo, municipalities Santo André and São Paulo capital (the latter for guide one and two). Ecuador: Salesian schools of a fiscal-private nature in the city of Cuenca. Spain: public and private schools of Cantabria. Italy: public schools in Rome and southern Italy. Mexico: public schools in Mexico City and metropolitan area. Uruguay: public and private schools of Montevideo.

The main result of this phase consisted of the agreement of the definitive version for the main study, made up of 21 questions and 11 deepening resources (e.g. what do you think other teachers would answer?) In a trilingual version. Three examples of such questions are presented in the table 3.

The results obtained were analyzed by means of virtual collective meetings, identifying emerging preliminary categories. Such analysis consisted of the identification of critical aspects about the guide, performance by researchers, and operation of the interview at the local level, among them the use of the direct and indirect modality. Researchers in Ecuador and Uruguay reported not perceiving significant differences in the responses obtained between the two interview modalities.
In Ecuador it was observed that the indirect modality did not favor proximity with the interviewees, required in this context. On the other hand, according to the results obtained in Italy, this same strategy allowed for the collection of broader responses, avoiding distortions by a researcher outside the school context. However, it was decided to discard such modality (so it was not replicated in Spain and Mexico) based on two criteria:

- It did not contribute to obtaining comparable responses between countries. Being similar to an informal conversation, the level of depth was not always predictable based on the arguments faced by the interviewer.

- The interviewees tended to answer questions directly, without expressing appreciation for the guide.

An outstanding aspect of piloting concerns the way in which the researchers, in their reading and analysis of the preliminary results, observed and interpreted the L2L competence according to the cultural perspective. From this situation it follows that capturing such representation is not an easy task; the recognition of the cultural traits of L2L by the researcher (or between researchers) and consequent discussion play a central role.

In this sense, the proposed categories (sense making, coping with the uncertainty and self-confidence), when evoking specific theoretical aspects, may or may not be part of the analytical view of the researchers, since they have different positions in this re-

Table 3.

Trilingual version of the final interview (questions 9, 16 and 17)

\begin{tabular}{|c|c|c|c|c|}
\hline Core thematic & Italian & Spanish & Portuguese & English \\
\hline \multirow{2}{*}{$\begin{array}{l}\text { Characteristics } \\
\text { of students that } \\
\text { announce success or } \\
\text { failure } \\
\text { (question 9) }\end{array}$} & \multirow{2}{*}{$\begin{array}{l}\text { Con il suo occhio da docente, } \\
\text { quali sono le caratteristiche } \\
\text { di uno studente che secondo } \\
\text { lei preannunciano successo } \\
\text { o insuccesso in futuro? }\end{array}$} & $\begin{array}{l}\text { De acuerdo con tu mirada } \\
\text { docente, ¿cuáles son las ca- } \\
\text { racterísticas de un }\end{array}$ & \multirow{2}{*}{$\begin{array}{l}\text { De acordo com seu 'olhar } \\
\text { docente', quais são as carac- } \\
\text { terísticas que indicam se um } \\
\text { aluno terá sucesso ou fracas- } \\
\text { so, no futuro? }\end{array}$} & $\begin{array}{l}\text { According to your teaching } \\
\text { vision, what are the charac- } \\
\text { teristics of a }\end{array}$ \\
\hline & & $\begin{array}{l}\text { estudiante que anuncian el } \\
\text { éxito o el fracaso en el futu- } \\
\text { ro? }\end{array}$ & & $\begin{array}{l}\text { student announcing success } \\
\text { or failure in the future? }\end{array}$ \\
\hline $\begin{array}{l}\text { Keep learning } \\
\text { (question 16) }\end{array}$ & $\begin{array}{l}\text { Secondo lei che cosa è ne- } \\
\text { cessario fare in modo che } \\
\text { gli studenti continuino ad } \\
\text { imparare? }\end{array}$ & $\begin{array}{l}\text { En tu opinión, ¿qué crees } \\
\text { que es necesario para garan- } \\
\text { tizar que los alumnos conti- } \\
\text { nuen aprendiendo? }\end{array}$ & $\begin{array}{l}\text { De acordo com você, o que } \\
\text { é necessário fazer para que } \\
\text { os alunos continuem apren- } \\
\text { dendo? }\end{array}$ & $\begin{array}{l}\text { In your opinion, what do } \\
\text { you think is necessary to } \\
\text { guarantee that students con- } \\
\text { tinue learning? }\end{array}$ \\
\hline $\begin{array}{l}\text { L2L (explicit) } \\
\text { (question 17) }\end{array}$ & $\begin{array}{l}\text { Se dovesse sintetizzarlo in } \\
\text { qualche modo, come carat- } \\
\text { terizzerebbe lei l'apprendere } \\
\text { ad apprendere, l'imparare a } \\
\text { imparare? }\end{array}$ & $\begin{array}{l}\text { Si tuvieras que sintetizarlo } \\
\text { de alguna manera, ¿cómo } \\
\text { caracterizarías el aprender a } \\
\text { aprender? }\end{array}$ & $\begin{array}{l}\text { Se você tivesse que de al- } \\
\text { guma maneira sintetizar o } \\
\text { aprender a aprender, como } \\
\text { você o faria? }\end{array}$ & $\begin{array}{l}\text { If you have to synthesize } \\
\text { in some way, how would } \\
\text { you characterize learning to } \\
\text { learn? }\end{array}$ \\
\hline
\end{tabular}


gard. Such diversity is considered inherent to research of a qualitative and intercultural nature (Creswell, 2016). The interpretations provided by the researchers around the guide represent conceptions associated with their own culture, their theoretical approach and their own notion of L2L. Rather than being avoided, such interpretations have been fundamental in the discussion and construction of a common object of study and methodology.

\section{Conclusive aspects}

The main contributions of this article consist of the exposition of the cultural perspective of L2L and presentation of the thematic structure of the interview. Both aspects represent innovation not only regarding how to obtain data that allows structuring the cultural map around this competence; it also means a complementary research framework to the approach of the predominant L2L studies.

The definition of a common instrument for the six countries, in turn, represents the achievement of understanding and coordinated work between researchers. Through this, it has been possible to intersect the repertoire of research practices of the participating teams. On the other hand, we consider that the development of the project and consequent systematization of data have the potential to benefit educational research in the following aspects:

- Identification and dissemination of teaching practices around the teaching of L2L from a cultural and daily point of view.

- Support for teacher training and design of educational policies to develop L2L competence from a perspective consistent with the local culture.

- Construction of definitions and methodologies arppro-priate to the educational contexts where this competence is to be fostered.

- Development of other research instruments focused on the knowledge of the cultural connotation of L2L.

Within the limitations of the article, it is necessary to consider the brief description of the interview and the respective topics. As a result, the restrictions associated with the scope of the instrument have not been addressed. In this sense, it is highlighted that the limits of the work adhere to the scope of a general approach around the process of design, translation and adaptation of the interview. It is considered necessary to discuss such aspects in depth at some other venue.

The following steps point to the analysis of the data from the main study and consolidation of international work between participating countries. In the medium term, the data are expected to shed light on the components through which L2L is culturally characterized, extending the potential application of the interview to other educational contexts. The final considerations of this article emphasize the need to invest efforts and resources in research of this nature, involving the expertise and knowledge of researchers in different countries. This will contribute to professional exchange and will create opportunities for discussion and discovery based on common problems in each educational reality, thereby promoting the identification and inclusion of their singularities and specificities.

\section{Acknowledgment}

We thank Anna Maria Ajello for her contribution in the design of the interview and Cristina Stringher for coordinating the project. Special thanks to all participating researchers.

\section{Referencias / References}

Aguiar, W., y Davis, C. (2011). Sentidos e significados no contexto escolar. Linguagens, educacao e sociedade (UFPI), 16(25), 183-196. Disponible en http://www.ufpi.br/subsiteFiles/ ppged/arquivos/files/Revista/les25.pdf

Ajello, A. (2018). L'imparare a imparare come competenza chiave nella societá globle. ValueEnews. 1, 3-5. Disponible en http:// adiscuola.it/limparare-a-imparare-come-competenza-chiave-nella-societa-globale/

Ajello, A., y Torti, D. (2019). Imparare a imparare come competenza chiave di cittadinanza e come soft skill. Scuola democratica, learning for democracy. 1, 63-82. doi:10.12828/93392

Álvarez-Arregui, E. (2019). Evolución de la Universidad en la Sociedad del Aprendizaje y la Enseñanza. El valor de las competencias en el desarrollo profesional y personal. Aula Abierta, 48(4), 349-372.

Arreguit, X. y Hugues, J. F. (2019). Competencias y educación para los trabajos y desafíos del mañana: La perspectiva de una empresa. Aula Abierta, 48(4), 373-392.

Bruner, J. (1990). Actos de significado: más allá de la revolución cognitiva. Madrid: Alianza Editorial.

Bruner, I. S. (1996). The culture of education. Cambridge: Harvard University Press.

Claxton, G. (2002). Education for the learning age: A sociocultural approach to learning to learn. In: G. Wells, y C. Claxton, (Eds.), Learning for life in the 21st Century: Sociocultural perspectives on the future of education (pp. 21-33). Oxford: Blackwell.

Comisión Europea (2006). Recomendación de 18 de Diciembre 2006 sobre las competencias clave para el aprendizaje permanente. Diario Oficial de la Unión Europea L 394/10 del 30.12.2006. Bruselas: Consejo Europeo. https://eur-lex.europa.eu/LexUriServ/LexUriServ.do?uri=OJ:L:2006:394:0010:0018:ES:PDF

Comisión Europea (2018). Council Recommendation of 22 May 2018 on Key Competences for LifeLong Learning. 2018/C 189/01-13. Brussels: European Council. https://eur-lex. europa.eu/legalcontent / EN / TXT / PDF / ?uri=CELEX:32018H0604(01)\&rid=7

Creswell, I. (2016). 30 essential skills for the qualitative researcher. London: Sage.

Deakin Crick, R., Broadfoot, P. y Claxton, G. (2004). Developing an effective lifelong learning inventory: the ELLI project. Assessment in Education, 11(3), 248-272.

Deakin Crick, R., Stringher, C., y Ren, K. (Eds.). (2014). Learning to learn. International Perspectives from Theory and Practice. Londres: Routledge.

Elshout-Mohr, M., Meijer, I., Oostdam, R., y Van Gelderen, A. (2004). CCST: A Test for CrossCurricular Skills. Amsterdam: University of Amsterdam.

Gardner, H. (1983). Frames of mind: The theory of multiple intelligences. New York: Basic Books.

Gargallo López, B.; Pérez-Pérez, C.; Garcia-Garcia, F.J.; Giménez Beut, I.A., y Portillo Poblador, N. (2020). La competencia aprender a aprender en la universidad: propuesta de modelo teórico. Educación XX1, 23(1), 19-44, doi: 10.5944/ educXX1.23367

Hager, P., y Halliday, I. (2006). Recovering Informal Learning. Wisdom, Judgment and Community. Dordrecht: Springer.

Harkness J., Bilgen I., Córdova A., Huang L., Miller D., Stange M. y Villar A. (2016). Questionnaire Design. Guidelines for Best Practice in Cross-Cultural Surveys. University of Michigan, Michigan. Disponible en: http://www.ccsg.isr.umich.edu/

Hautamäki J., Arinen P., Eronen S., Hautamäki A., Kupiainen S., Lindblom B., Niemivirta M., Pakaslahti L., Rantanen P. y 
Scheinin P. (2002), Assessing Learning-to-learn. A Framework. Helsinki: Helsinki University printing house.

Hautamäki, I., y Kupiainen, S. (2014). Learning to learn in Finland. Theory and policy, research and practice. En R. Deakin Crick, C. Stringher y K. Ren (Eds.), Learning to learn International perspective from theory and practice (pp. 170-195). Londres: Routledge.

Heckman, I., Stixrud, J. y Urzúa S. (2006). The effects of cognitive and noncognitive abilities on labor market outcomes and social behavior. Journal of Labor Economics. 24(3), 411-482.

Kvale, S. (2011). Las entrevistas en investigación cualitativa. Madrid: Ediciones Morata.

Larkin, P. I., de Casterlé, B., y Schotsmans, P. (2007). Multilingual translation issues in qualitative research: Reflections on a metaphorical process. Qualitative Health Research, 17(4), 468476. https:/ / doi.org/10.1177/1049732307299258.

Losito G. (1988). Metodi e tecniche della ricerca sociale empirica sull'emittenza. En M. Livolsi M. Y F. Rositi (Eds.). La ricerca sull'industria culturale (pp. 31-55). Roma: NIS.

Marcuccio, M. (2016). Imparare a imparare nei contesti scolastici. Prospettive e sfide per l'innovazione didattica. Roma: Armando editore.

Mauceri, S. (2018). Mixed survey research. Le funzioni delle tecniche qualitative nella ricerca standard. Sociologia e ricerca sociale, 116, 90-102, doi:10.3280/sr2018-116008

Montesperelli, P. (1998). L'intervista ermeneutica. Milán: FrancoAngeli.

OECD (2009). Informe PISA 2009. Aprendiendo a aprender. Vol. III. Madrid: OCDE. Disponible en https://www.oecd-ilibrary. org/education/informe-pisa-2009-aprendiendo-a-aprender 9789264177529-es.

Patera, S. (2018). Learning to learn in Latin America: A quali-quantitative comparative analysis of national curricoli and educational systems of compulsory education in Latin America. Working Paper INVALSI, 32/2018, 1-56.

Rogoff, B. (1990). Apprenticeship in thinking: Cognitive development in social context. Oxford University Press.
Roth, W. (2013). Translation in Qualitative Social Research: The Possible Impossible [49 paragraphs]. Forum Qualitative Sozialforschung / Forum: Qualitative Social Research, 14(2), Art.13 http://nbnresolving.de/urn:nbn:de:0114-fqs1302132.

Santos, B. de S. (2011). Epistemologías del Sur. Utopía y Praxis Latinoamericana. 6 (54),17-39.

Serranò, F. y Fasulo, A. (2011). L'intervista come conversazione. Preparazione, conduzione e analisi del colloquio di ricerca. Roma: Carocci.

Stringher, C. (2014). What is learning to learn? A learning to learn process and output model. En R. Deakin Crick, C. Stringher, $\mathrm{K}$. Ren (Eds.), Learning to learn. International perspectives from theory and practice (pp. 9-40). Londres: Routledge.

Stringher, C., Di Rienzo, P., Brito, H., Davis, C., y García, E. (2019). Aprender a aprender en América Latina. Una reseña sistemática de la literatura. En R. Deakin Crick, C. Stringher y K. Ren (Eds.), Aprender a aprender. Perspectivas internacionales, desde la teoría y la práctica (pp. 357-368). México: Trillas.

Szymanski, H., Almeida, L., y Prandini, R. (2004). A entrevista na pesquisa em educacão: a prática reflexiva. Brasília: Liber Livro.

Temple, B., y Young, A. (2004). Qualitative research and translation dilemmas. Qualitative Research, 4 (2), 161-178. https:// doi.org/10.1177/1468794104044430.

Trinchero, R. (2002). Manuale di ricerca educativa. Milán: Franco Angeli.

Tuomi-Gröhn, T., y Engeström, Y. (2003). Conceptualizing transfer: From standard notions to developmental perspectives. En T. Tuomi-Gröhn y. Y. Engeström (Eds.), Between school and work: New perspectives on transfer and boundary crossing (pp. 19-38). Amsterdam: Pergamon Press.

UNESCO (2013). Toward Universal Learning: What Every Child Should Learn. Reports No. 1. Sin lugar: UNESCO.

Van Ness, F., Abma, T., Jonsson, H., y Deeg, D. (2010). Language differences in qualitative research: Is meaning lost in translation? European Journal of Ageing., 7, 313-316. doi:10.1007/ s10433-010-0168-y

Vygotsky, L.S. (1986). Thought and language. Cambridge: Massachusetts Institute of Technology Press. 
